(ACE). The knowledge gained from this pilot shows that it is feasible to recruit, train and utilise the unique skills of patients as volunteers to support other (newly diagnosed) patients to accept their chronic, life changing condition, make informed choices about their treatment while empowering them.

We have the proof of principle that PEs can meet the needs of renal units, working within the NHS Trust volunteer/ governance framework. This initiative is low cost, demonstrating new ways of working and managing, empowering patients and utilizing non -clinical workforce through effective leadership.

\section{IMPROVING HEALTH OUTCOMES BY A SOUTH LONDON LOOKED AFTER CHILDREN'S (LAC) TEAM}

Stacy John-Legere*, Abby Sinclair. Evelina London, Guys and St. Thomas NHS Foundation Trust, UK

\subsection{6/leader-2018-FMLM.102}

Context There is an obligation on the local authority and its health partners to ensure these statutory health assessments take place in accordance with the timescales set. The looked after children's health team delivers this responsibility on behalf the local authority. A significant portion live outside the borough boundaries. The multidisciplinary team comprises specialist nurses, community paediatricians and administrative team.

Issue Timely and thorough health assessments are vehicles for identifying and acting upon previously unmet health needs in this vulnerable population.

Assessment of issue and analysis of its causes The Change Management Framework

The ADKAR ${ }^{\circledR}$ model of change was utilised. The team were aware of the need for change.The trust executive and management had a desire to support and participate in the change. We utilised the knowledge of the core team and additional expertise. We have also established a system of reinforcement to sustain the change.

The strategy:

To deliver on the changes needed a clear leadership strategy was in place.

- It was purpose driven

- Previous inspection reports, user feedback and performance indicators were used

- Clear and constant two-way communication was employed

o weekly performance huddles, and

- Investment in people and infrastructure occurred

$\circ$ job plans developed, and increased training and development opportunities given

- We demonstrated persistence to see the changes through

- Improvements were shared on the ground at team meetings, at directorate level; and at Trust executive level. The changes were shared with the Service Improvement Board, the CCG Safeguarding executive and the local authority.

Measurement of improvement and impact:

- In March 2018 100\% of children accessing initial assessments were seen within 20 working days from referral; the graph (figure 1) shows the overall trend of improvement

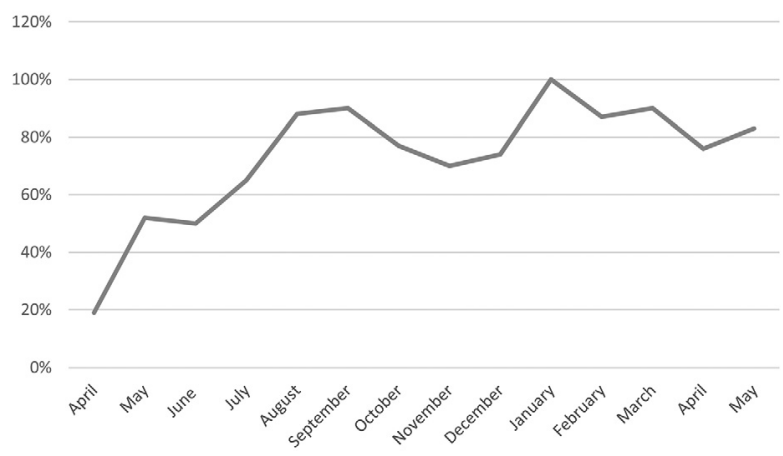

Abstract 104 Figure 1\% of children accessing initial assessments within 20 working days of referral- April 2017 to May 2018

- There is now knowledge of where all LAC are in the pathway, including those that are being seen out of borough and those that are in secure accommodation

- Improvement in staff morale measured by a fully established team, reduction in staff turnover rate and sickness levels

Impact The next steps are:

- Sustaining cultural and process changes in both the clinical and administrative team

- Continue to work with social care to improve the referral process

- Ensuring systematic oversight of the completion of health recommendations arising from assessment.

\section{DOCTOR (NOUN, LATIN), TO TEACH; TRANSFORMING A WORKING WEEK INTO A TEACHING WEEK}

${ }^{1}$ Ahmed Chilmeran*, ${ }^{2}$ Alexandros Rampotas, ${ }^{1}$ Mustafa Agbna, 'Sathianathan Panthakalam. ${ }^{1}$ East Sussex Healthcare NHS Trust, UK; ${ }^{2}$ Oxford Health NHS Foundation Trust, UK

\subsection{6/leader-2018-FMLM.103}

One of the prerequisites to successfully complete Core Medical Training in the UK is to acquire the MRCP title by passing the Practical Assessment of Clinical Examination Skills (PACES). Due to work pressures and time limitations, little teaching had been taking place locally at Eastbourne District General Hospital (EDGH). Prior to this, PACES teaching was mainly delivered externally by expensive courses, online resources and books. Given the plethora of patients in a familiar environment of an NHS hospital, we established that this provided an ideal combination for PACES teaching.

We started a team of PACES positive trainees who were interested in teaching; this team would deliver bedside PACES teaching at least twice a week. A weekly timetable with specified PACES teaching was created (e.g table 1). This included some specialised clinics that were consultant led (rheumatology and ophthalmology) aimed at providing consultant led teaching to PACES candidates. One PACES candidate was allocated on each clinic day providing 1-to-1 focused teaching, while the bedside sessions were open for everyone. A WhatsApp group was also created consisting of all candidates preparing for PACES and instructors, which encouraged all members to share interesting cases deemed good for PACES teaching and also facilitated organising unscheduled teaching. 